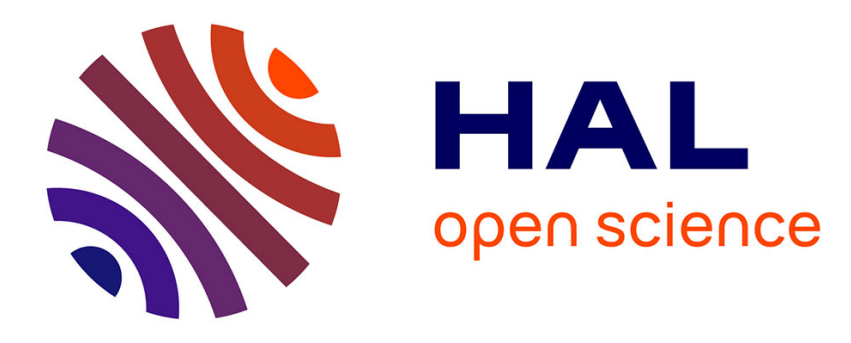

\title{
New bounds on the Grundy number of products of graphs
}

Victor Campos, Andras Gyarfas, Frédéric Havet, Claudia Linhares Sales, Frédéric Maffray

\section{- To cite this version:}

Victor Campos, Andras Gyarfas, Frédéric Havet, Claudia Linhares Sales, Frédéric Maffray. New bounds on the Grundy number of products of graphs. Journal of Graph Theory, 2012, 71 (1), pp.7888. hal-00749190

\author{
HAL Id: hal-00749190 \\ https://hal.inria.fr/hal-00749190
}

Submitted on 23 Oct 2016

HAL is a multi-disciplinary open access archive for the deposit and dissemination of scientific research documents, whether they are published or not. The documents may come from teaching and research institutions in France or abroad, or from public or private research centers.
L'archive ouverte pluridisciplinaire HAL, est destinée au dépôt et à la diffusion de documents scientifiques de niveau recherche, publiés ou non, émanant des établissements d'enseignement et de recherche français ou étrangers, des laboratoires publics ou privés. 


\title{
New bounds on the Grundy number of products of graphs*
}

\author{
Victor Campos ${ }^{\dagger} \quad$ András Gyárfás ${ }^{\ddagger} \quad$ Frédéric Havet $^{\S}$ \\ Claudia Linhares Sales ${ }^{\Uparrow} \quad$ Frédéric Maffray"
}

September 8, 2010

\begin{abstract}
The Grundy number of a graph $G$ is the largest $k$ such that $G$ has a greedy $k$ colouring, that is, a colouring with $k$ colours obtained by applying the greedy algorithm according to some ordering of the vertices of $G$. In this paper, we give new bounds on the Grundy number of the product of two graphs.
\end{abstract}

\section{Introduction}

Graphs considered in this paper are undirected, finite and contain neither loops nor multiple edges (unless stated otherwise). The definitions and notations used in this paper are standard and may be found in any textbook on graph theory; see [4] for example. Given two graphs $G$ and $H$, the direct product $G \times H$, the lexicographic product $G[H]$, the Cartesian product $G \square H$ and the strong product $G \otimes H$ are the graphs with vertex set $V(G) \times V(H)$ and the following edge sets:

$$
\begin{aligned}
E(G \times H) & =\{(a, x)(b, y) \mid a b \in E(G) \text { and } x y \in E(H)\} ; \\
E(G[H]) & =\{(a, x)(b, y) \mid \text { either } a b \in E(G) \text { or } a=b \text { and } x y \in E(H)\} ; \\
E(G \square H) & =\{(a, x)(b, y) \mid \text { either } a=b \text { and } x y \in E(H) \text { or } a b \in E(G) \text { and } x=y\} ; \\
E(G \otimes H) & =E(G \times H) \cup E(G \square H) .
\end{aligned}
$$

* Research supported by CAPES-COFECUB project MA 622/08 and the INRIA Equipe Associée EWIN.

${ }^{\dagger}$ Dept. of Computer Science, Federal University of Ceará, Fortaleza, CE, Brazil. campos@lia.ufc.br.

${ }^{\ddagger}$ Computer and Automation Research Institute, Hungarian Academy of Sciences, Budapest, P.O. Box 63, Budapest, Hungary, H-1518, gyarfas@sztaki.hu. Research supported in part by OTKA Grant No. K68322.

${ }^{\S}$ Projet Mascotte, I3S (CNRS, UNSA) and INRIA, 2004 route des lucioles, BP 93, 06902 Sophia-Antipolis Cedex, France. fhavet@sophia.inria.fr. Partly supported by ANR Blanc AGAPE.

`Dept. of Computer Science, Federal University of Ceará, Fortaleza, CE, Brazil. linhares@lia.ufc.br

"CNRS, Laboratoire G-SCOP, Grenoble, France. frederic.maffray@inpg.fr. 
A $k$-colouring of a graph $G$ is a surjective mapping $\psi: V(G) \rightarrow\{1, \ldots, k\}$. It is proper if for every edge $u v \in E(G), \psi(u) \neq \psi(v)$. A proper $k$-colouring may also be seen as a partition of the vertex set of $G$ into $k$ disjoint non-empty stable sets (i.e. sets of pairwise non-adjacent vertices) $C_{i}=\{v \mid \psi(v)=i\}$ for $1 \leq i \leq k$. For convenience (and with a slight abuse of terminology), by proper $k$-colouring we mean either the mapping $\psi$ or the partition $\left\{C_{1}, \ldots, C_{k}\right\}$. The elements of $\{1, \ldots, k\}$ are called colours. A graph is $k$ colourable if it admits a $k$-colouring. The chromatic number $\chi(G)$ is the least $k$ such that $G$ is $k$-colourable.

Many upper bounds on the chromatic number arise from algorithms that produce colourings. The most basic one is the greedy algorithm. A greedy colouring relative to a vertex ordering $v_{1}<v_{2}<\cdots<v_{n}$ of $V(G)$ is obtained by colouring the vertices in the order $v_{1}, \ldots, v_{n}$, assigning to $v_{i}$ the smallest positive integer not already used on its lowerindexed neighbours. Trivially, a greedy colouring is proper. Denoting by $C_{i}$ the stable set of vertices coloured $i$, a greedy colouring has the following property:

For every $i<j$, every vertex in $C_{j}$ has a neighbour in $C_{i}$,

for otherwise the vertex in $C_{j}$ would have been coloured $i$ or less. Conversely, a colouring satisfying Property $(\star)$ is a greedy colouring relative to any vertex ordering in which the vertices of $C_{i}$ precede those of $C_{j}$ whenever $i<j$. The Grundy number $\Gamma(G)$ is the largest $k$ such that $G$ has a greedy $k$-colouring.

Let $\Delta(G)$ denote the maximum degree in a graph $G$. Let $K_{n}$ denote the complete graph on $n$ vertices and $K_{p, q}$ denote the complete bipartite graph with parts of size $p$ and $q$. Let $S_{n}$ denote the edgeless graph on $n$ vertices.

In [1], Asté, Havet and Linhares Sales investigated the Grundy number of several types of graph products. They showed that the Grundy number of the lexicographic product of two graphs is bounded in terms of the Grundy numbers of these graphs.

Theorem 1 ([1]). For any two graphs $G$ and $H, \Gamma(G[H]) \leq 2^{\Gamma(G)-1}(\Gamma(H)-1)+\Gamma(G)$.

Moreover, when the graph $G$ is a tree, they obtained an exact value.

Theorem 2 ([1]). Let $T$ be a tree and $H$ be any graph. Then $\Gamma(T[H])=\Gamma(T) \Gamma(H)$.

They also showed that, in contrast with the lexicographic product, there is no upper bound of $\Gamma(G \square H)$ as a function of $\Gamma(G)$ and $\Gamma(H)$; for example, $\Gamma\left(K_{p, p}\right)=2$ and $\Gamma\left(K_{p, p} \square K_{p, p}\right) \geq p+1$. Nevertheless, they showed that $\Gamma(G \square H)$ is bounded by a function of $\Delta(G)$ and $\Gamma(H)$.

Theorem 3 ([1]). For any two graphs $G$ and $H, \Gamma(G \square H) \leq \Delta(G) \cdot 2^{\Gamma(H)-1}+\Gamma(H)$.

However, they conjectured that this upper bound is far from being tight.

Conjecture 4 ([1]). For any two graphs $G$ and $H, \Gamma(G \square H) \leq(\Delta(G)+1) \Gamma(H)$.

This conjecture generalises the following conjecture of Balogh, Hartke, Liu and Yu [3]. 
Conjecture 5 ([3]). For any graph $H, \Gamma\left(K_{2} \square H\right) \leq 2 \Gamma(H)$.

Here is another conjecture that would imply the preceding one.

Conjecture 6 (Havet and Zhu). If $G$ is any graph and $M$ is a matching in $G$, then $\Gamma(G) \leq 2 \Gamma(G \backslash M)$.

In [7], Havet, Kaiser and Stehlik proved Conjecture 4 in the case when one of $G, H$ is a tree.

Theorem 7 ([7]). For any graph $G$ and tree $T, \Gamma(G \square T) \leq(\Delta(G)+1) \Gamma(T)$.

Here we investigate further the relation between the Grundy number of the direct product, lexicographic product or Cartesian product of two graphs and the invariants $\Gamma$ and $\Delta$ of the two graphs. We first show that $\Gamma(G \square H) \leq \Gamma\left(H\left[K_{\Delta(G)+1}\right]\right)$. Together, with Theorem 1 and 2, this implies Theorems 3 and 7 respectively. In particular, we obtain a shorter proof of Theorem 7 .

We then show that $\Gamma\left(G\left[K_{2}\right]\right)=\Gamma\left(G\left[S_{2}\right] \square K_{2}\right)$. As a corollary, we give an example of a graph that disproves Conjectures 4,5 and 6: there is a graph $H$ such that $\Gamma(H)=3$ and $\Gamma\left(K_{2} \square H\right)=7$. Together with Theorem 3 this yields $\max \left\{\Gamma\left(K_{2} \square H\right) \mid \Gamma(H)=3\right\}=7$.

Regarding the direct and strong product, we answer a question raised as the last sentence in [1]. There cannot be any bound on $\Gamma(G \times H)$ and $\Gamma(G \otimes H)$ as a function of $\Gamma(G), \Gamma(H)$ if $\Gamma(G), \Gamma(H) \geq 3$ (Theorem 15). It is also impossible to bound $\Gamma(G \times H)$ in terms of $\Delta(G), \Gamma(H)$ when $G$ is any graph with at least one edge and $\Gamma(H) \geq 5$ (Theorems 17). Similarly, it is impossible to bound $\Gamma(G \otimes H)$ in terms of $\Delta(G), \Gamma(H)$ when $\Gamma(H) \geq 5$ unless $G$ is the disjoint union of complete graphs (Theorem 18 and Proposition19).

\section{The Cartesian and lexicographic products}

\subsection{Common proof of Theorems 3 and 7}

Theorem 8. For any two graphs $G$ and $H, \Gamma(G \square H) \leq \Gamma\left(G\left[K_{\Delta(H)+1}\right]\right)$.

Proof. We shall prove that if $G \square H$ has a greedy $q$-colouring for some integer $q$, then so does $G\left[K_{\Delta(H)+1}\right]$. Hence consider a greedy $q$-colouring $\varphi$ of $G \square H$. Let $(x, y)$ be a vertex of $G \square H$ with colour $q$. Let $x_{1}, x_{2}, \ldots, x_{n}$ be an ordering of the vertices of $G$ such that $\varphi\left(x_{1}, y\right) \leq \varphi\left(x_{2}, y\right) \leq \cdots \leq \varphi\left(x_{n}, y\right)$.

Let us denote by $z_{0}, \ldots, z_{\Delta(H)}$ the vertices of $K_{\Delta(H)+1}$. For every $1 \leq i \leq n$, we assign colour $\varphi\left(x_{i}, y\right)$ to vertex $\left(x_{i}, z_{0}\right)$ of $G\left[K_{\Delta(H)+1}\right]$. Then for $i=1$ to $n$, we do the following. Let $L_{i}$ be the set of colours less than $\varphi\left(x_{i}, y\right)$ that have not been assigned to any neighbour of $\left(x_{i}, z_{0}\right)$ in $G\left[K_{\Delta(H)+1}\right]$. Since $\varphi$ is a greedy colouring and colour $\varphi\left(x_{j}, y\right)$ is assigned to $\left(x_{j}, z_{0}\right)$ for each $j, L_{i}$ is a subset of $\left\{\varphi\left(x_{i}, u\right) \mid u \in N(y)\right\}$. Therefore $\left|L_{i}\right| \leq \Delta(H)$. Hence we can assign all the colours of $L_{i}$ to distinct vertices in $\left\{\left(x_{i}, z_{j}\right) \mid 1 \leq j \leq \Delta(H)\right\}$. 
Let us show that the obtained partial $q$-colouring of $G\left[K_{\Delta(H)+1}\right]$ is a greedy colouring. It is proper since colours already assigned to neighbours of $\left(x_{i}, z_{0}\right)$ are not in $L_{i}$. In $L_{i}$ we add every colour $\ell<\varphi\left(x_{i}, z_{0}\right)$ such that $\left(x_{i}, z_{0}\right)$ had no neighbour coloured $\ell$ before Step $i$. Hence, after Step $i$, vertex $\left(x_{i}, z_{0}\right)$ has a neighbour of each colour less than $\varphi\left(x_{i}, y\right)$. Now every coloured vertex $\left(x_{i}, z\right)$ has a colour $\ell$ less than $\varphi\left(x_{i}, y\right)$. But, by the definition of the lexicographic product, all neighbours of $\left(x_{i}, z_{0}\right)$, except $\left(x_{i}, z\right)$ itself, are neighbours of $\left(x_{i}, z\right)$. Hence $\left(x_{i}, z\right)$ has a neighbour of each colour less than $\ell$. So the colouring is greedy.

\subsection{Disproof of Conjecture 4}

Asté, Havet and Linhares Sales [1] proved the following:

Lemma 9 ([1]). For any graph $G$ and any integer $n, \Gamma\left(G\left[S_{n}\right]\right)=\Gamma(G)$.

Now we prove:

Theorem 10. Let $G$ be a graph. Then $\Gamma\left(G\left[K_{2}\right]\right)=\Gamma\left(G\left[S_{2}\right] \square K_{2}\right)$.

Proof. Let us show that the left hand side is at most the right hand side. Consider a greedy colouring $\varphi$ of $G\left[K_{2}\right]$. Every vertex $v$ of $G$ corresponds to two adjacent vertices of $G\left[K_{2}\right]$. Let us denote by $\varphi_{1}(v)$ and $\varphi_{2}(v)$ the two distinct colours assigned by $\varphi$ to these vertices. In the graph $G\left[S_{2}\right] \square K_{2}$, every vertex $v$ corresponds to four vertices $a_{v}$, $b_{v}, a_{v}^{\prime}$ and $b_{v}^{\prime}$ inducing two edges $a_{v} b_{v}$ and $a_{v}^{\prime} b_{v}^{\prime}$, and so that if $u v$ is any edge of $G$, then $G\left[S_{2}\right] \square K_{2}$ has all edges between $\left\{a_{u}, a_{u}^{\prime}\right\}$ and $\left\{a_{v}, a_{v}^{\prime}\right\}$ and all edges between $\left\{b_{u}, b_{u}^{\prime}\right\}$ and $\left\{b_{v}, b_{v}^{\prime}\right\}$. Assign colour $\varphi_{1}(v)$ to $a_{v}$ and $b_{v}^{\prime}$ and colour $\varphi_{2}(v)$ to $b_{v}$ and $a_{v}^{\prime}$. Doing this for every vertex, it is easy to check that we obtain a greedy colouring of $G\left[S_{2}\right] \square K_{2}$. Hence $\Gamma\left(G\left[K_{2}\right]\right) \leq \Gamma\left(G\left[S_{2}\right] \square K_{2}\right)$.

Let us now show that the right hand side is at most the left hand side. By Theorem 8 , we have $\Gamma\left(G\left[S_{2}\right] \square K_{2}\right) \leq \Gamma\left(G\left[S_{2}\right]\left[K_{2}\right]\right)$. We claim that $\Gamma\left(G\left[S_{2}\right]\left[K_{2}\right]\right) \leq \Gamma\left(G\left[K_{2}\right]\right)$. To see this, consider any greedy colouring $\varphi$ of $G\left[S_{2}\right]\left[K_{2}\right]$ with $q$ colours. In $G\left[S_{2}\right]\left[K_{2}\right]$, every vertex $v$ of $G$ corresponds to four vertices $a_{v}, b_{v}, c_{v}, d_{v}$ with two edges $a_{v} b_{v}, c_{v} d_{v}$, and for every edge $u v$ of $G$, there are all edges between $\left\{a_{u}, b_{u}, c_{u}, d_{u}\right\}$ and $\left\{a_{v}, b_{v}, c_{v}, d_{v}\right\}$. Suppose that $\varphi$ assigns at least three different colours in $\left\{a_{v}, b_{v}, c_{v}, d_{v}\right\}$ for some $v$, say $\varphi\left(a_{v}\right)=i$, $\varphi\left(b_{v}\right)=j, \varphi\left(c_{v}\right)=k$, where, up to symmetry, $i<j$ and $k \notin\{i, j\}$. Note that $b_{v}$ has no neighbour of colour $k$, because its neighbours are either $a_{v}$ or adjacent to $c_{v}$. So $j<k$. At least one colour $h \in\{i, j\}$ is not the colour of $d_{v}$, so $c_{v}$ has no neighbour of colour $h$, a contradiction. So $\varphi$ uses exactly two colours in $\left\{a_{v}, b_{v}, c_{v}, d_{v}\right\}$ for every vertex $v$ of $G$. It follows that the restriction of $\varphi$ on the subgraph of $G\left[S_{2}\right]\left[K_{2}\right]$ induced by $\left\{a_{v}, b_{v} \mid v \in V(G)\right\}$, which is isomorphic to $G\left[K_{2}\right]$, is a greedy colouring with $q$ colours. So the claim that $\Gamma\left(G\left[S_{2}\right] \square K_{2}\right) \leq \Gamma\left(G\left[K_{2}\right]\right)$ is established. This completes the proof.

Remark 11. Theorem 10 can be generalised in a straightforward manner to the following result: Let $G$ be any graph and $p$ be any integer. Then $\Gamma\left(G\left[K_{p}\right]\right)=\Gamma\left(G\left[S_{p}\right] \square K_{p}\right)$. 
Theorem 10 implies that Conjectures 4, 5 and 6 do not hold, as follows.

Corollary 12. There is a graph $H$ such that $\Gamma(H)=3$ and $\Gamma\left(K_{2} \square H\right)=7$.

Proof. Let $G_{3}$ be the graph that consists of a cycle of length 6 plus one vertex $g$ adjacent to a vertex $a$ of the cycle and one vertex $h$ adjacent to another vertex $b$ of the cycle, where $a$ and $b$ are adjacent. Let $H=G_{3}\left[S_{2}\right]$. Asté, Havet and Linhares Sales [1] showed that $\Gamma\left(G_{3}\right)=3$ and $\Gamma\left(G_{3}\left[K_{2}\right]\right)=7$. Hence, Lemma 9 yields $\Gamma(H)=3$ and Theorem 10 yields $\Gamma\left(K_{2} \square H\right)=7$. This proves the corollary.

Alternately, let $G_{3}^{\prime}$ be the graph obtained from $G_{3}$ by identifying the two vertices $g$ and $h$ (i.e., replacing them by one vertex adjacent to $a$ and $b$ ), and let $H^{\prime}=G_{3}^{\prime}\left[S_{2}\right]$. Then one can also check that $\Gamma\left(H^{\prime}\right)=3$ and $\Gamma\left(K_{2} \square H^{\prime}\right)=7$.

Clearly, the two graphs $H$ and $H^{\prime}$ mentioned in the preceding proof are counterexamples to Conjectures 4 and 5. Note also that if $v$ is any vertex of $H$ and $a_{v}, b_{v}$ are the corresponding two vertices in $K_{2} \square H$, then the set $M=\left\{a_{v} b_{v} \mid v \in V(H)\right\}$ is a matching in $K_{2} \square H$, and $\left(K_{2} \square H\right) \backslash M$ consists of two disjoint copies of $H$ with no edge between them; so $\Gamma\left(\left(K_{2} \square H\right) \backslash M\right)=3$. This shows that $K_{2} \square H$ is a counterexample to Conjecture 6 . The same holds for $K_{2} \square H^{\prime}$.

Corollary 12 shows that Conjecture 4 does not hold if $\Gamma(H)=3$. On the other hand, we now show that Conjecture 4 holds if $\Gamma(H)=2$.

Proposition 13. Let $G$ and $H$ be two graphs. If $\Gamma(H)=2$ then $\Gamma(G \square H) \leq 2(\Delta(G)+1)$.

Proof. If $\Gamma(H)=2$ then $H$ is a complete bipartite graph [10]. Let $(A, B)$ be its bipartition. For every vertex $v \in V(G)$, define $A_{v}=\{(v, a) \mid a \in A\}$ and $B_{v}=\{(v, b) \mid b \in B\}$, so $A_{v}$ and $B_{v}$ are the two sides of the copy of $H$ indexed by $v$ in $G \square H$. Let $\varphi$ be a greedy colouring of $G \square H$. We claim that:

$$
\text { For any } v \in V(G),\left|\varphi\left(A_{v}\right)\right| \leq \Delta(G)+1 \text { and }\left|\varphi\left(B_{v}\right)\right| \leq \Delta(G)+1 \text {. }
$$

Assume for a contradiction, and up to symmetry, that $\left|\varphi\left(A_{v}\right)\right| \geq \Delta(G)+2$. Let $\alpha$ be the largest colour of $\varphi\left(A_{v}\right)$ and let $x=(v, a)$ be a vertex coloured $\alpha$. The neighbourhood of $x$ in $G \square H$ is $B_{v} \cup\left\{(w, a) \mid w \in N_{G}(v)\right\}$. But the colours of $\varphi\left(A_{v}\right)$ do not appear on $B_{v}$ because it is complete to $A_{v}$, and $\left|\left\{(w, a) \mid w \in N_{G}(v)\right\}\right|=d_{G}(v) \leq \Delta(G)$. Hence at most $\Delta(G)$ colours of $\varphi\left(A_{v}\right)$ may appear on the neighbourhood of $x$, and so at least one colour of $\varphi\left(A_{v}\right) \backslash\{\alpha\}$ does not. This contradicts the fact that $\varphi$ is a greedy colouring and proves the claim.

Let $y=(v, b)$ be a vertex such that $\varphi(y)$ is maximum. Without loss of generality, we may assume that $b \in B$. At most $2 \Delta(G)+1$ colours appear in the neighbourhood of $y$ : at most $\Delta(G)+1$ on $A_{v}$ according to the claim, and at most one more for each of its neighbours not in $B_{v}$, whose number is $d_{G}(y) \leq \Delta(G)$. Hence $\varphi(y) \leq 2 \Delta(G)+2$. 
Remark 14. Proposition 13 can easily be generalised to complete multipartite graphs in a straightforward manner to obtain the following result: if $H$ is a complete multipartite graph, then $\Gamma(G \square H) \leq(\Delta(G)+1) \Gamma(H)$.

\section{The direct and strong products}

Here we show that $\Gamma(G \times H)$ and $\Gamma(G \otimes H)$ cannot be bounded by a function of $\Gamma(G)$ and $\Gamma(H)$ if $\Gamma(G), \Gamma(H) \geq 3$ (Theorem 15 ). It is also a natural question to bound $\Gamma(G \times H)$ or $\Gamma(G \otimes H)$ in terms of $\Delta(G)$ and $\Gamma(H)$. For $\Delta(G)=1$, a non-trivial construction of [2] shows that $3\lceil\Gamma(H) / 2\rceil-1 \leq \Gamma\left(K_{2} \times H\right)$. Somewhat surprisingly, we show in Theorem 17 that there is no upper bound on $\Gamma\left(K_{2} \times H\right)$ in terms of $\Gamma(H)$ if $\Gamma(H) \geq 5$. Moreover, we show in Theorem 18 that there is no upper bound on $\Gamma\left(P_{3} \otimes H\right)$ in terms of $\Gamma(H)$ if $\Gamma(H) \geq 5$. In fact, Theorem 18 implies that there is no upper bound on $\Gamma(G \otimes H)$ as a function $\Delta(G)$ and $\Gamma(H)$ for $\Gamma(H) \geq 5$ unless $G$ is the disjoint union of complete graphs. In Proposition 19, we show that there is an upper bound in such a case.

Let us first recall some definitions. The binomial tree is the graph $T_{k}$ defined recursively as follows. For $k=1, T_{1}$ is the one-vertex graph. For $k \geq 2, T_{k}$ is obtained from $T_{k-1}$ by adding, for each vertex $v$ of $T_{k-1}$, one vertex $v^{\prime}$ with an edge $v v^{\prime}$. It is easy to see that, for $k \geq 2, T_{k}$ has two adjacent vertices $r, s$ of degree $k-1$ and the other vertices have degree at most $k-2$, and the two components of $T_{k} \backslash r s$ are both isomorphic to $T_{k-1}$. We view $T_{k}$ as rooted at vertex $r$. We have $\Gamma\left(T_{k}\right)=k$. More precisely, $T_{k}$ has a greedy colouring $\psi$ where each vertex $v \notin\{r, s\}$ has colour equal to its degree, and $s, r$ have colour $k-1$ and $k$ respectively. Note that for each vertex $v$ and colour $i<\psi(v), v$ has a unique neighbour of colour $i$.

The radius of a graph $G$ is the smallest integer $t$ for which there exists a vertex $a$ of $G$ such that every vertex of $G$ is at distance at most $t$ from $a$. Note that the radius of $T_{k}$ is $k-1$. It is easy to see that every tree with radius at most 2 has Grundy number at most three. This is also a corollary of the following result from $[5,6]$ : the Grundy number of a tree is equal to the Grundy number of its largest binomial subtree, and of the fact that the radius of a subtree of a tree $T$ is not larger than the radius of $T$.

Theorem 15. For every $k \geq 3$, there is a graph $G$ such that $\Gamma(G)=3$ and $\Gamma(G \times G) \geq k$ and $\Gamma(G \otimes G) \geq k$.

Proof. Let $G$ be the graph obtained from $T_{k}$ by subdividing every edge once. Partition the vertex set of $G$ into two stable sets $A$ and $B$ such that $A$ contains the original vertices of $T_{k}$ and $B$ contains the subdivision vertices. Consider any greedy colouring of $G$. Every vertex in $B$ has degree two and consequently receives a colour from the set $\{1,2,3\}$. Moreover, a vertex in $B$ receives colour 3 if and only if its two neighbours have received colours 1 and 2 respectively. It follows that no vertex of $A$ can receive colour 4 or more. This implies that $\Gamma(G) \leq 3$. Since $G$ contains a four-vertex path, $\Gamma(G) \geq 3$. Thus $\Gamma(G)=3$. To complete 
the proof of the theorem, let us show that $G \times G$ and $G \otimes G$ have a common induced subgraph $H_{k}$ isomorphic to $T_{k}$. This implies $\Gamma(G \times G) \geq k$ and $\Gamma(G \otimes G) \geq k$.

Let the root $r$ of $T_{k}$ become the root of $G$. Since $G$ is viewed as a rooted tree, every vertex in $B$ has one parent and one child. Consider the greedy colouring $\psi$ of $T_{k}$ with $k$ colours as defined above, such that the root $r$ has colour $k$ and the second vertex $s$ of degree $k-1$ has colour $k-1$. For $i \in\{1, \ldots, k\}$, let $A_{i}$ be the set of vertices in $A$ that receive colour $(k+1)-i$. So $A_{1}=\{r\}$ and $A_{2}=\{s\}$. For each $i \in\{2, \ldots, k\}$, let $B_{i}$ be the set of vertices in $B$ whose child is in $A_{i}$. We say that a vertex $v$ in $A_{i} \cup B_{i}$ has label $i$ and denote by $\ell_{v}$ the label of $v$. Let $q$ be the parent of $s$ (i.e., $q$ is the common neighbour of $r$ and $s$ ). Let $d(x, y)$ denote the distance between any two vertices $x$ and $y$ in $G$. We prove by induction on $i \in\{2, \ldots, k\}$ that $G \times G$ and $G \otimes G$ have an induced subgraph $H_{i}$ such that:

(1) $H_{i}$ is isomorphic to $T_{i}$ and contains vertex $(r, q)$.

(2) Every vertex of $H_{i}$ is of the form $(a, b)$ or $(b, a)$, with $a \in A$ and $b \in B$; moreover, $\ell_{a}<\ell_{b} \leq i$, vertices $a, b$ lie in distinct components of $G \backslash r q$, and $d(a, r)=d(b, q)$.

For $i=2$, the induced subgraph $H_{2}$ with vertices $(r, q)$ and $(q, r)$ and an edge between them is the desired copy of $T_{2}$. Now let $i \geq 3$. By the induction hypothesis, there exists a common induced subgraph $H_{i-1}$ of $G \times G$ and $G \otimes G$ that satisfies (1) and (2). Let $z$ be any vertex of $H_{i-1}$, and let $a \in A$ and $b \in B$ be such that $z$ is equal to $(a, b)$ or $(b, a)$. Let $u$ be the unique child of $b$ in $G$. By the definition of the labels we have $\ell_{u}=\ell_{b}$. By property (2), we have $\ell_{a} \leq i-1$, so (in $T_{k}$, and since $\psi$ is a greedy colouring) $a$ has a neighbour of colour $(k+1)-i$, and (in $G$ ) $a$ has a neighbour $v \in B$ with label $i$. Clearly, $u$ and $v$ lie in distinct components of $G \backslash r q$ since $a$ and $b$ do. Now, either $(v, u)$ or $(u, v)$ is a neighbour of $(a, b)$ in $G \times G$ and we call this neighbour the leaf of $z$, and $z$ is called the support of its leaf. Note that any leaf-support edge is also an edge in $G \otimes G$ as $E(G \times G) \subseteq E(G \otimes G)$. Since $v$ has label $i$, the leaf of $z$ is not a vertex in $H_{i-1}$. Since $\ell_{u}=\ell_{b} \leq i-1$ and $\ell_{v}=i$, we have $\ell_{u}<\ell_{v} \leq i$. Since $u$ is a child of $b$ and $v$ is a child of $a$, we have $d(u, r)=d(v, q)$. (More precisely: if $a$ lies in the component $G_{r}$ of $G \backslash r q$ that contains $r$ and $b$ lies in the other component $G_{q}$, then $d(u, r)=d(b, q)+2$ and $d(v, q)=d(a, r)+2$; if on the contrary $a$ lies in $G_{q}$ and $b$ lies in $G_{r}$, then $d(u, r)=d(b, q)$ and $d(v, q)=d(a, r)$.)

Let $V_{i-1}$ be the vertex set of $H_{i-1}$ and let $W_{i-1}$ be the set of leaves of vertices in $V_{i-1}$. Let $H_{i}$ be the subgraph of $G \otimes G$ induced by the vertices in $V_{i-1} \cup W_{i-1}$. As observed above, $H_{i}$ satisfies property (2). In order to show that $H_{i}$ is isomorphic to $T_{i}$, we need only prove that (i) each vertex in $W_{i-1}$ has a unique neighbour in $V_{i-1}$ and (ii) $W_{i-1}$ induces a stable set. Note that this also implies that $H_{i}$ is an induced subgraph in $G \times G$ as $E(G \times G) \subseteq E(G \otimes G)$.

To show that Claim (i) is true, suppose on the contrary that the leaf $(v, u) \in W_{i-1}$ of some vertex $(a, b) \in V_{i-1}$ is adjacent to a vertex $(x, y) \in V_{i-1}$ different from $(a, b)$. Up to symmetry we may assume that $a, u \in A$ and $b, v \in B$ and that $a$ lies in $G_{r}$ and $b$ in $G_{q}$ 
(the argument in the other cases is similar). We must have $x=a$, for otherwise $x$ is either $v$ or the child of $v$ and $\ell_{x}=i$, which contradicts property (2) in $H_{i-1}$. Since $x \in A$, then $y \in B$ by property (2). Now, $y \neq b$, and $y$ is a child of $u$. Now $d(y, q)=d(b, q)+2$, whereas $d(x, r)=d(a, r)$, so $d(x, r) \neq d(y, q)$, a contradiction.

To show that Claim (ii) is true, suppose on the contrary that $(a, b)$ and $\left(b^{\prime}, a^{\prime}\right)$ are two adjacent vertices in $W_{i-1}$. We can consider $a, a^{\prime} \in A$ and $b, b^{\prime} \in B$ as they could not be adjacent otherwise. Let $\left(s_{a}, s_{b}\right)$ and $\left(s_{b^{\prime}}, s_{a^{\prime}}\right)$ be the supports of $(a, b)$ and $\left(b^{\prime}, a^{\prime}\right)$, respectively. Note that $s_{a}, s_{a^{\prime}} \in B$ and $s_{b}, s_{b^{\prime}} \in A$, which implies that $\ell_{s_{b}}<\ell_{s_{a}}$ and $\ell_{s_{b^{\prime}}}<\ell_{s_{a^{\prime}}}$. By the definition of the labels, we have $\ell_{s_{a}}=\ell_{a}$ and $\ell_{s_{a^{\prime}}}=\ell_{a^{\prime}}$. Moreover, each of $b$ and $b^{\prime}$ has label $i$ and consequently has a child of label $i$ and $\ell_{a}<\ell_{b}=i$. Thus, for $(a, b)$ to be adjacent to $\left(b^{\prime}, a^{\prime}\right), a$ must be the neighbour of $b^{\prime}$ with label smaller than $i$, which is $s_{b^{\prime}}$. In particular, $\ell_{a}=\ell_{s_{b^{\prime}}}$, and, by a symmetric argument, $\ell_{a^{\prime}}=\ell_{s_{b}}$. Putting this all together, we obtain that if $(a, b)$ is adjacent to $\left(b^{\prime}, a^{\prime}\right)$, then $\ell_{a}=\ell_{s_{b^{\prime}}}<\ell_{s_{a^{\prime}}}=\ell_{a^{\prime}}=\ell_{s_{b}}<\ell_{s_{a}}=\ell_{a}$ which is a contradiction.

To prove Theorem 17 and Theorem 18, we study the graph $H_{k}$ defined as follows. We start from the binomial tree $T_{k}$ whose vertex set is partitioned into three sets $X_{1}, X_{2}, X_{3}$. The root of $T_{k}$ is in $X_{1}$. For every $v \in X_{1} \cup X_{3}$, the children of $v$ are in $X_{2}$. For every $v \in X_{2}$ the children of $v$ are placed according to the position of the parent $w$ of $v$ : if $w \in X_{1}$ then the children of $v$ are in $X_{3}$; if $w \in X_{3}$ then the children of $v$ are in $X_{1}$. Now $H_{k}$ is obtained by adding to $T_{k}$ all edges between $X_{1}$ and $X_{3}$.

Theorem 16. For $k \geq 1, \Gamma\left(H_{k}\right) \leq 5$. Furthermore, for $k \geq 9, \Gamma\left(H_{k}\right)=5$.

Proof. We first observe that $\Gamma\left(H_{k}\right) \leq 6$ for every $k$. Indeed, in $H_{k}$ every stable set is contained either in $A_{1}=X_{1} \cup X_{2}$ or in $A_{2}=X_{2} \cup X_{3}$. If $H_{k}$ admits a greedy colouring with at least seven colours, then at least four colour classes are included in one of the two sets $A_{1}$ and $A_{2}$, say in $A_{j}$. This means that the subgraph $H^{*}$ induced by $A_{j}$ in $H_{k}$ has Grundy number at least four. However, each component of $H^{*}$ is a tree of radius at most two, which implies that $H^{*}$ has Grundy number at most three.

In order to complete the first part of the theorem, let us give a more detailed analysis to show that $\Gamma\left(H_{k}\right) \leq 5$. The following two properties of $T_{k}$ are useful.

(1) Any vertex $v \in X_{2}$ has either exactly one neighbour in $X_{1}$ or exactly one neighbour in $X_{3}$ (because if the parent of $v$ is in one of $X_{1}, X_{3}$, then all its children are in the other of these two sets).

(2) For $i=1,3$, no path on five vertices in $X_{i} \cup X_{2}$ has its two endvertices in $X_{i}$ (because every component of $X_{i} \cup X_{2}$ consists of either the root of $T_{k}$ and its children, or some vertex of $X_{2}$, its children and its grandchildren.)

Suppose that there exists a greedy 6-colouring $\varphi$ on $H_{k}$.

Case 1: $\varphi(v) \in\{5,6\}$ for $v \in X_{2}$. Vertex $v$ has neighbours of colours 1,2,3,4. By property (1), $v$ is adjacent to at most one vertex of $X_{1}$ or $X_{3}$. So there is $i \in\{1,3\}$ such 
that $v$ has neighbours $w_{1}, w_{2}, w_{3} \in X_{i}$ with $\varphi\left(w_{1}\right)<\varphi\left(w_{2}\right)<\varphi\left(w_{3}\right) \leq 4$. Then $w_{3}$ has a neighbour $w_{4}$ with $\varphi\left(w_{4}\right)=\varphi\left(w_{2}\right)$, and $w_{4}$ has a neighbour $w_{5}$ with $\varphi\left(w_{5}\right)=\varphi\left(w_{1}\right)$. Since $\left\{w_{2}, w_{4}\right\}$ and $\left\{w_{1}, w_{5}\right\}$ are stable sets, we have $w_{4} \in X_{2}$ and $w_{5} \in X_{i}$. But then the path $w_{1}-v-w_{3}-w_{4}-w_{5}$ contradicts property $(2)$.

Case 2: $\varphi(v)=6$ for some $v \in X_{1} \cup X_{3}$. Let $i$ be the index in $\{1,3\}$ such that $v \in X_{i}$. Vertex $v$ has a neighbour $w$ with $\varphi(w)=5$. Then $w \in X_{4-i}$, otherwise Case 1 applies. Vertices $v$ and $w$ have neighbours $u_{v}$ and $u_{w}$ of colour 4 , possibly $u_{v}=u_{w}$, but we cannot have one in $X_{1}$ and the other in $X_{3}$. Hence one vertex $u \in\left\{u_{v}, u_{w}\right\}$ is in $X_{2}$. Let $t$ be its neighbour in $\{v, w\}$ and $j$ the index such that $t \in X_{j}$. Vertex $u$ has three neighbours $a, b, c$ such that $\{\varphi(a), \varphi(b), \varphi(c)\}=\{1,2,3\}$. By property (1), either two elements of $\{a, b, c\}$, say $a, b$, are in $X_{j}$, or $\{a, b, c\} \subset X_{4-j}$. If $a, b \in X_{j}$, we may assume $\varphi(a)<\varphi(b)$, and we pick a neighbour $d$ of $t$ with $\varphi(d)=\varphi(b)$ and a neighbour $e$ of $d$ with $\varphi(e)=\varphi(a)$. Since $\{a, e\}$ and $\{b, d\}$ are stable sets in $H_{k}$, we have $d \in X_{2}, e \in X_{j}$. But then the path $e$ - $d$-t-u-a contradicts property (2). If $\{a, b, c\} \subset X_{4-j}$, we may assume that $\varphi(a)=1, \varphi(b)=2$ and $\varphi(c)=3$. There is a neighbour $d$ of $c$ with $\varphi(d)=2$ and a neighbour $e$ of $d$ with $\varphi(e)=1$. Since $\{a, e\}$ and $\{b, d\}$ are stable sets in $H_{k}$, we have $d \in X_{2}, e \in X_{4-j}$. But then the path $e$ - $d$-c- $u$ - $a$ contradicts property (2). Thus we have shown that $\Gamma\left(H_{k}\right) \leq 5$, which completes the first part of the theorem.

Now, we show that $\Gamma\left(H_{k}\right)=5$ when $k \geq 9$. We know that $\Gamma\left(T_{k}\right)=k$, so $T_{k}$ contains a path $a_{1}-a_{2} \cdots-a_{9}$ whose vertices are coloured $k, k-1, \ldots, k-8$ respectively, where $a_{1}$ is the root of $T_{k}$, and a path $a_{2}-b_{3}-b_{4}-b_{5}$ whose vertices are coloured $k-1, k-3, k-4, k-5$, and a path $a_{6}-b_{7}-b_{8}$ whose vertices are coloured $k-5, k-7, k-8$. Note that vertices $a_{1}, a_{5}, a_{9}, b_{5}$ are in $X_{1}$, vertices $a_{2}, a_{4}, a_{6}, a_{8}, b_{4}, b_{8}$ are in $X_{2}$ and vertices $a_{3}, a_{7}, b_{3}, b_{7}$ are in $X_{3}$. Now we can make a greedy colouring of $H_{k}$ with five colours, where vertices $a_{2}, a_{5}, b_{5}, b_{8}, a_{9}$ receive colour 1 , vertices $a_{3}, b_{4}, b_{7}, a_{8}$ receive colour 2 , vertices $b_{3}, a_{6}$ receive colour 3 , and vertices $a_{1}$ and $a_{7}$ receive colours 4 and 5 .

Theorem 17. If $G$ is a graph with at least one edge and $k \geq 1$, then $\Gamma\left(G \times H_{k}\right) \geq k$.

Proof. It is enough to prove the theorem when $G=K_{2}, V(G)=\left\{v_{1}, v_{2}\right\}$. We claim that $\Gamma\left(G \times H_{k}\right) \geq k$. To see this, let $Y_{i}=\left\{v_{1}\right\} \times X_{i}$ for $i=1,3$ and $Y_{2}=\left\{v_{2}\right\} \times X_{2}$. Then it is easy to check that $Y_{1} \cup Y_{2} \cup Y_{3}$ induces a copy of $T_{k}$ in $K_{2} \times H_{k}$, where $Y_{i}$ plays the role of $X_{i}$ in the partition of $H_{k}$.

Theorem 18. If $G$ is a connected non-complete graph and $k \geq 1$, then $\Gamma\left(G \otimes H_{k}\right) \geq k$.

Proof. It is enough to prove the theorem when $G=P_{3}=v_{1}-v_{2}-v_{3}$ as $G$ contains an induced subgraph isomorphic to $P_{3}$. We claim that $\Gamma\left(G \otimes H_{k}\right) \geq k$. To see this, let $Y_{i}=\left\{v_{i}\right\} \times X_{i}$ for $i \in\{1,2,3\}$. It is easy to check that $Y_{1} \cup Y_{2} \cup Y_{3}$ induces a copy of $T_{k}$ in $P_{3} \otimes H_{k}$, where $Y_{i}$ plays the role of $X_{i}$ in the partition of $H_{k}$.

If $G$ is a the disjoint union of complete graphs, then there is an upper bound on $\Gamma(G \otimes H)$ as a function of $\Gamma(G)$ and $\Gamma(H)$. It is enough to consider the case $G=K_{m+1}$. Observe 
that $K_{m+1} \otimes H=H\left[K_{m+1}\right]$. Hence by Theorem 1 we get the following.

Proposition 19. If $\Gamma(H)=k \geq 2$ and $m \geq 1$ then $\Gamma\left(K_{m+1} \otimes H\right) \leq m 2^{k-1}+k$.

\section{Comments and open questions}

Section 3 shows that any upper bound on the Grundy number of $G \times H$ as a function of $\Delta(G), \Gamma(H)$ is possible only if $\Gamma(H) \leq 4$. Perhaps a good test case is to decide whether $\Gamma\left(K_{2} \times H\right)$ is bounded for $\Gamma(H) \leq 4$. (On the other hand, if the maximum degree of both graphs may intervene, then we know the easy inequality $\Gamma(G \times H) \leq \Delta(G \times H)+1 \leq$ $\Delta(G) \Delta(H)+1$, but this is probably not a very interesting bound.)

Concerning the lexicographic product, it was proved in [1] that if $\Gamma(H)=k$, then for any graph $G$, we have $\Gamma(G[H])=\Gamma\left(G\left[K_{k}\right]\right)$. Moreover, as mentioned in Remark 11, we have $\Gamma\left(G\left[K_{k}\right]\right)=\Gamma\left(G\left[S_{k}\right] \square K_{k}\right)$. So $\Gamma(G[H])=\Gamma\left(G\left[S_{k}\right] \square K_{k}\right)$. Thus the Grundy number of the lexicographic product of any two graphs $G$ and $H$ can be seen as a particular case of the Grundy number of the Cartesian product of two graphs. Therefore we feel that the most interesting questions in this domain are about the Cartesian product. In particular, although Conjecture 4 is now known to be false because of Corollary 12, one may still wonder whether there exists a constant $\lambda$ such that any two graphs $G$ and $H$ satisfy $\Gamma(G \square H) \leq \lambda(\Delta(G)+1) \Gamma(H)$. Note that the graph $H$ given in the proof of Corollary 12 gives the ratio $\Gamma\left(K_{2} \square H\right) /\left\{\left(\Delta\left(K_{2}\right)+1\right) \Gamma(H)\right\}=7 / 6$, and the second graph $H^{\prime}$ gives the same ratio. We could not find a graph with a larger ratio. Is it true that $\Gamma\left(K_{2} \square H\right) \leq c \Gamma(H)$ for some constant $c \geq 7 / 6$ ?

\section{References}

[1] M. Asté, F. Havet, C. Linhares Sales. Grundy number and products of graphs. Discrete Mathematics 310:1482-1490, 2010.

[2] M. Asté, F. Havet, C. Linhares Sales. Grundy number and products of graphs. INRIA Research Report R-6672, 2008.

[3] J. Balogh, S.G. Hartke, Q. Liu, G. Yu. On the first-fit chromatic number of graphs. SIAM J. Discrete Math. 22(3):887-900, 2008.

[4] J. A. Bondy, U. S. R. Murty. Graph theory. Graduate Texts in Mathematics 244, Springer, 2008.

[5] A. Gyárfás, J. Lehel. First-fit and on-line chromatic number of families of graphs. Ars Combinatoria 29C:160-167, 1990.

[6] A. Gyárfás, Z. Király, J. Lehel. On-line graph colouring and finite basis problems. Combinatorics, Paul Erdős is eighty, Bolyai Math. Studies 1:207-214, 1993. 
[7] F. Havet, T. Kaiser, M. Stehlik. Grundy number of the Cartesian product of a tree and a graph. Unpublished.

[8] S.M. Hedetniemi, S.T. Hedetniemi, A. Beyer. A linear algorithm for the Grundy (colouring) number of a tree. Congr. Numer. 36:351-363, 1982.

[9] D.G. Hoffman, P.D. Johnson Jr. Greedy colourings and the Grundy chromatic number of the $n$-cube. Bull. Inst. Combin. Appl. 26:49-57, 1999.

[10] M. Zaker. Results on the Grundy chromatic number of graphs. Discrete Math. 306(23):3166-3173, 2006. 